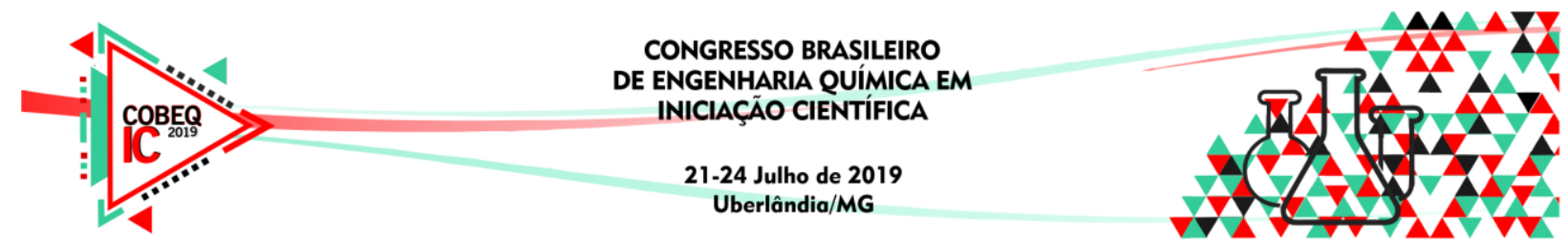

\title{
INFLUENNCIA DE DIFERENTES CORPOS INERTES NA DINÂMICA DE PARTÍCULAS EM TAMBOR ROTATÓRIO UTILIZANDO ABORDAGEM LAGRANGEANA
}

\author{
O. F. S. NETTO ${ }^{1}$, R. L. SANTOS $^{1}$, C. R. DUARTE ${ }^{1}$ e M. A. S. BARROZO ${ }^{1}$ \\ ${ }^{1}$ Universidade Federal de Uberlândia, Faculdade de Engenharia Química \\ E-mail para contato: oscarfonseca94@gmail.com
}

\begin{abstract}
RESUMO - O secador rotatório é utilizado na indústria por apresentar baixos custos de operação, simplicidade de construção e flexibilidade na secagem de diversos tipos de materiais. $\mathrm{O}$ uso de partículas inertes neste tipo de secador visa aumentar a eficiência do mesmo ao facilitar a exposição do material a ser seco pelo fluido de secagem. A atuação destas partículas depende, dentre outros fatores, das suas características físicas, como dimensão, forma, densidade e das condições em que o secador rotatório é operado. Este trabalho avaliou a influência do tipo de partícula, grau de preenchimento e da velocidade de rotação do tambor na dinâmica de esferas de aço e porcelana por meio do Método dos Elementos Discretos (DEM). Através das simulações realizadas, verificou-se que os inertes de aço apresentaram número de colisões, força e energia desprendida superiores àquelas verificadas para as de porcelana. Tais respostas podem ter reflexos em uma maior eficiência do secador, haja vista que o mecanismo de secagem e desprendimento do material seco tem relação direta com as colisões praticadas dentro do tambor. Além disso, essas respostas mostraram-se superiores com quantidades maiores de inertes dentro do tambor.
\end{abstract}

\section{INTRODUÇÃO}

A secagem pode ser descrita com um processo termodinâmico que visa à redução da atividade de água $\left(\mathrm{a}_{\mathrm{w}}\right)$ de um determinado meio. Este processo é importante na indústria, pois permite o prolongamento da vida útil de alimentos ao dificultar a proliferação de microorganismos por conta da ausência de água. Este processo está dentre os mais antigos métodos de conservação de alimentos por conta da sua simplicidade e baixo custo. Além disso, existem varias bases para a secagem, cada uma com suas características definidas pela natureza do alimento, sua forma e qualidade final desejada (Moura, 2016; Mujundar, 2006).

Os secadores rotatórios são empregados na indústria por conta do seu baixo custo operacional, por ser de fácil construção e por ser flexível quanto aos materiais a serem secos (Sheehan et al, 2005). A adição de corpos inertes ao secador contribui para a formação de partículas menores de pó seco, além de diminuir a decomposição de compostos orgânicos. No entanto, há a necessidade de estudos a respeito do comportamento de tais inertes dentro do sistema de secagem (Freire et al., 2012).

Para isso, o método dos elementos discretos (DEM) pode ser aplicado na análise do comportamento dinâmico deste material. Na aproximação DEM, são acompanhados os 


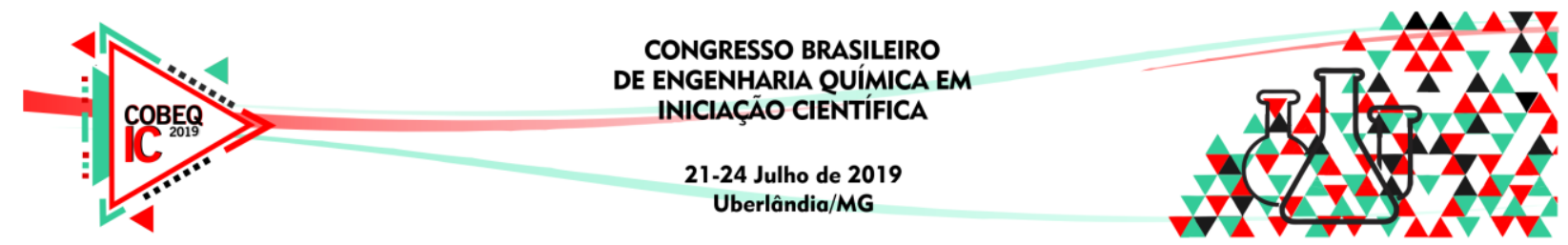

movimentos das partículas através da aplicação de uma integração temporal no balanço de forças sobre cada partícula individualmente, considerando inclusive as interações partículapartícula e partícula-parede (Brandão, 2017).

Este tipo de modelagem é regido basicamente por duas etapas principais: a lei de deslocamento (displacement law), que analisa as forças de contato partícula-partícula e partícula-parede; e a segunda Lei de Newton, que analisa a influência das forças de contato e de campo no movimento da partícula. A partir deste artifício, é possível determinar a aceleração da partícula por meio da razão entre a força resultante pela sua massa. Com isso, o algoritmo estima as trajetórias das partículas ao atualizar os valores de velocidade, deslocamento e rotação (PENG, 2014).

Para uma partícula $i$ com massa $m_{i}$ e momento de inércia $I_{i}$ dois tipos de força são, em geral, levados em consideração: a força peso, resultado do campo gravitacional, e a força de contato. Quando uma partícula $i$ colide com uma partícula j (ou parede j), a força de contato é decomposta em duas componentes: normal $\left(F_{c, n, i i}\right)$ e tangencial $\left(F_{c, t, i i}\right)$. A segunda Lei de Newton, então, pode ser escrita tal qual apresentado na Equação 1.

$$
m_{i} \frac{d v_{i}}{d t}=m_{i} g+\sum_{j=1}^{n_{i}}\left(F_{c, n, i j}+F_{\mathrm{c}, t, i j}\right)
$$

O modelo não linear para modelagem do contato entre partículas comumente utilizado no DEM é resultado da combinação da teoria clássica de contato elástico desenvolvida por Hertz (1882) para a direção normal, e o modelo de não deslizamento proposto por Mindlin \& Deresiewicz (1953) para a direção tangencial. De acordo com este modelo, a força normal, dada pela Equação 2, é função da sobreposição normal $\left(\delta_{n}\right)$, do raio de contato equivalente $\left(R^{*}\right)$ e do módulo de Young equivalente $\left(E^{*}\right)$. A força tangencial, por sua vez, descrita na Equação 3, é função da sobreposição tangencial $\left(\delta_{t}\right)$, módulo de cisalhamento equivalente $\left(G^{*}\right)$.

$$
\begin{aligned}
& F_{n}=\frac{4}{3} E^{*} \delta_{n}^{3 / 2} \sqrt{R^{*}} \\
& F_{t}=-8 G^{*} \delta_{t} \sqrt{R^{*} \delta_{n}}
\end{aligned}
$$

Assim, este trabalho visou investigar a dinâmica de partículas inertes em tambor rotatório com respeito ao tipo de partícula e a variáveis operacionais como grau de preenchimento (FD) e velocidade de rotação (RS) com auxílio do método DEM. As respostas avaliadas para tal fim foram número de colisões, força normal e energia desprendida durante as colisões.

\section{MATERIAL E MÉTODOS}

O tambor utilizado foi de aço inoxidável, com $12 \mathrm{~cm}$ de diâmetro e $36 \mathrm{~cm}$ de comprimento. O tambor possuía 3 suspensores (flights) de $2,5 \mathrm{~cm}$ de altura uniformemente dispostos na direção axial. A malha utilizada para representar a geometria nos cálculos DEM 


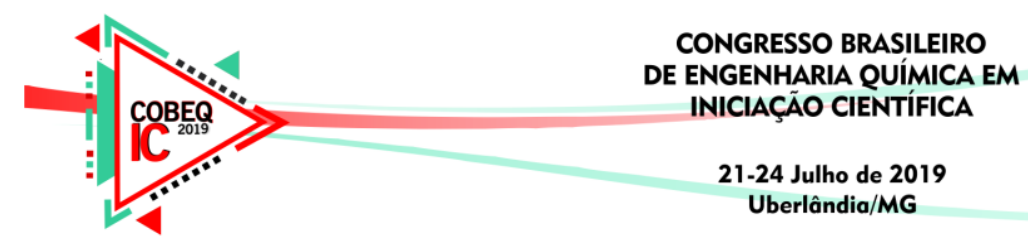

foi gerada pelo software Blender. Os inertes utilizados no estudo foram esferas de porcelana e de aço, ambas com $1,9 \mathrm{~cm}$ de diâmetro e densidades de $2320 \mathrm{~kg} / \mathrm{m}^{3}$ e $7800 \mathrm{~kg} / \mathrm{m}^{3}$, respectivamente.

Todas as simulações realizadas neste trabalho utilizaram o software EDEM $^{\circledR}$. Os valores dos parâmetros utilizados na simulação são apresentados na Tabela 1.

Tabela 1- Parâmetros utilizados na simulação

\begin{tabular}{|c|c|c|}
\hline Parâmetro & Valor (Aço) & Valor (Porcelana) \\
\hline Coeficiente de Restituição (-) & 0,6 & 0,9 \\
\hline Coeficiente de atrito estático (-) & 0,3 & 0,8 \\
\hline Coeficiente de atrito de rolamento (-) & 0,005 & 0,010 \\
\hline Razão de Poisson (-) & 0,3 & 0,2 \\
\hline Módulo de Young (N/m $\left.{ }^{2}\right)$ & $2,08 \times 10^{8}$ & $1 \times 10^{6}$ \\
\hline Time-Step (s) & $6 \times 10^{-5}$ & $1 \times 10^{-4}$ \\
\hline Tempo Simulado (s) & 60 & 60 \\
\hline
\end{tabular}

As condições utilizadas na simulação foram determinadas de acordo com o planejamento composto central apresentado na Tabela 2. Foram avaliadas as variáveis grau de preenchimento (FD) e velocidade de rotação (RS) do tambor. Quanto às respostas DEM, foram quantificados os números de colisões (entre partículas e partículas-parede), a força normal média de colisão e a energia perdida durante as colisões.

Tabela 2 - Planejamento composto central utilizado nas simulações

\begin{tabular}{|c|c|c|c|c|}
\hline \multirow{2}{*}{ Condição (S) } & \multicolumn{2}{|c|}{ Grau de preenchimento } & \multicolumn{2}{c|}{ Velocidade de rotação } \\
\cline { 2 - 5 } & Níveis (-) & $(\%)$ & Níveis (-) & (RPM) \\
\hline 1 & -1 & 15,0 & -1 & 40,0 \\
\hline 2 & -1 & 15,0 & +1 & 60,0 \\
\hline 3 & 1 & 27,0 & -1 & 40,0 \\
\hline 4 & 1 & 27,0 & +1 & 60,0 \\
\hline 5 & 0 & 21,0 & 0 & 50,0 \\
\hline 6 & $-1,483$ & 12,1 & 0 & 50,0 \\
\hline 7 & $+1,483$ & 29,9 & 0 & 50,0 \\
\hline 8 & 0 & 21,0 & $-1,483$ & 35,2 \\
\hline 9 & 0 & 21,0 & $+1,483$ & 64,8 \\
\hline 10 & $+1,483$ & 29,9 & $+1,483$ & 64,8 \\
\hline
\end{tabular}

\section{RESULTADOS OBTIDOS}

As figuras 1, 2 e 3 apresentam gráficos que relacionam o número de colisões das esferas de aço e porcelana dentro do tambor rotatório no decorrer do tempo. 


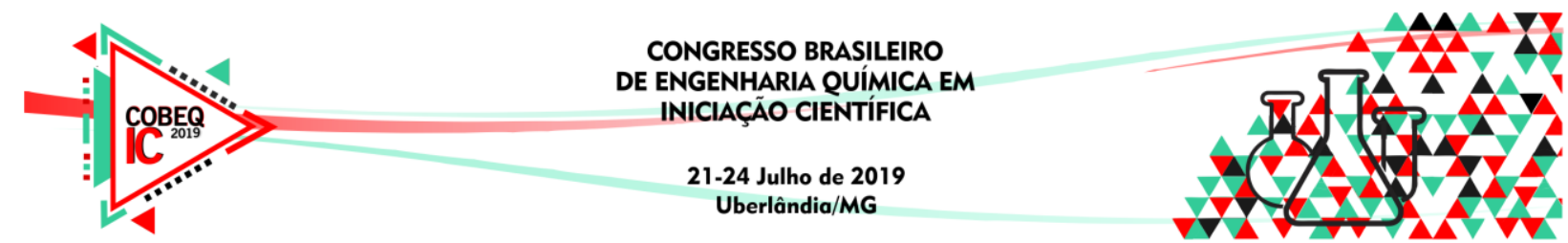

Figura 1 - Tambor com grau de preenchimento de 15\%: (a) 40 RPM e (b) 60RPM.
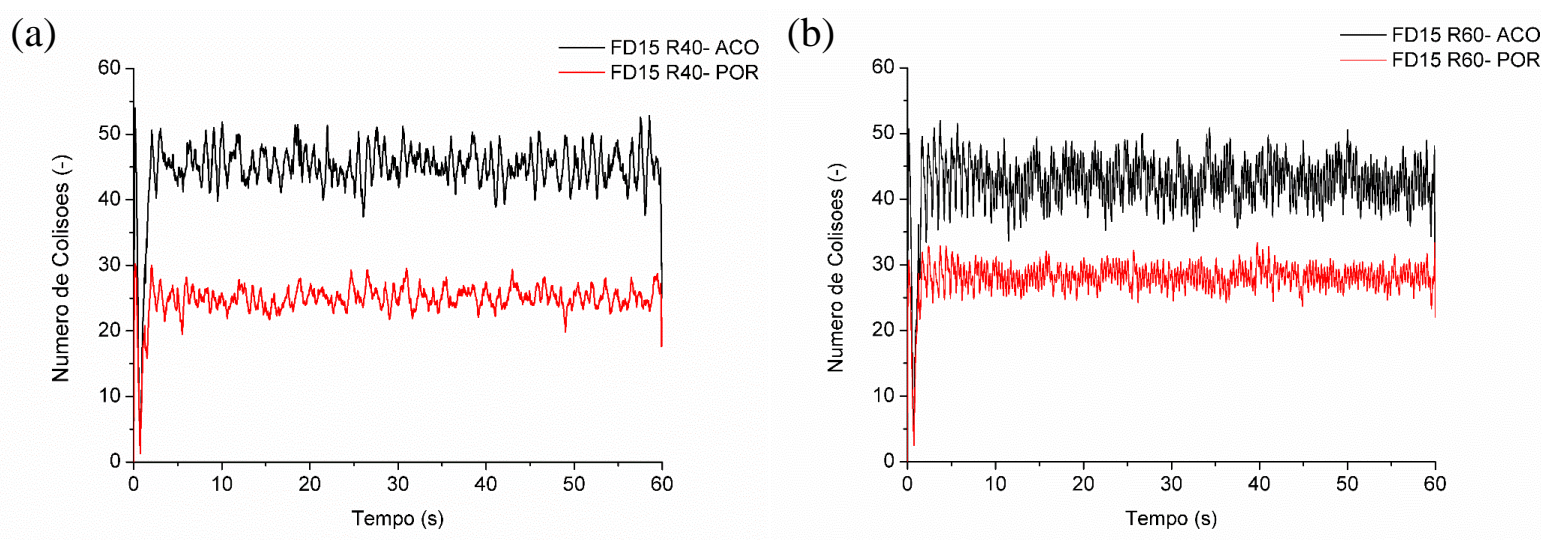

Figura 2 - Tambor com grau de preenchimento de 27\% a 40 RPM.

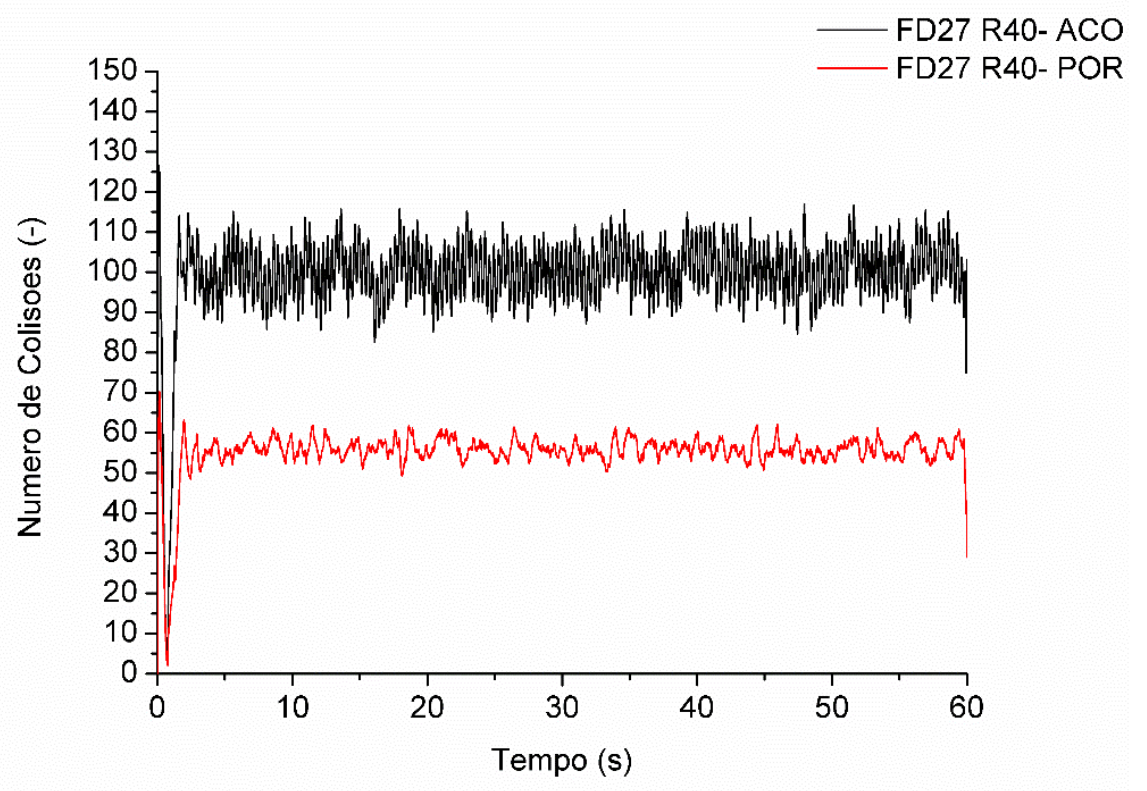

Como observado nas Figuras 1 e 2, o número de colisões ao longo do tempo em ambos os casos avaliados foi maior para as esferas de metal. Assim, as esferas de aço podem levar a um maior rendimento do material na secagem, pois o número de colisões atua no desprendimento da camada de material seco aderido tanto na superfície do inerte e quanto nas paredes internas do tambor (Burjaili, 1996).

Com respeito às Figuras 1a e 2, observou-se que houve um aumento expressivo do número de colisões de ambas as esferas com a alteração do grau de enchimento. Devido ao aumento no número de partículas dentro do tambor a ocorrência de interações partículapartícula e partícula-parede se torna maior.

A comparação realizada entre os dados apresentados nas Figuras 1a, 1b e 2 mostrou que o aumento do grau de enchimento foi de maior impacto em relação ao aumento da velocidade. 


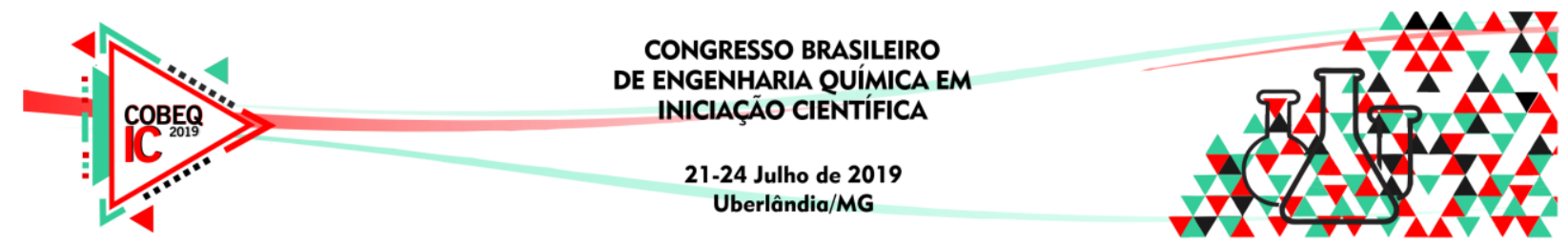

As Figuras 3 e 4 apresentam, respectivamente, gráficos que relacionam o número de colisões ponderadas (número de colisões por partícula) com a Força Normal Média de Colisão e com a Energia de Colisão das esferas de aço e porcelana. O uso do número de colisões ponderado pelo número de partículas de faz necessário para viabilizar a comparação entre simulações realizadas em condições de enchimento distintas (Bracey et al, 2016).

Figura 3 - Correlação obtida para Força Normal Média de Colisão: (a) Aço e (b) Porcelana.
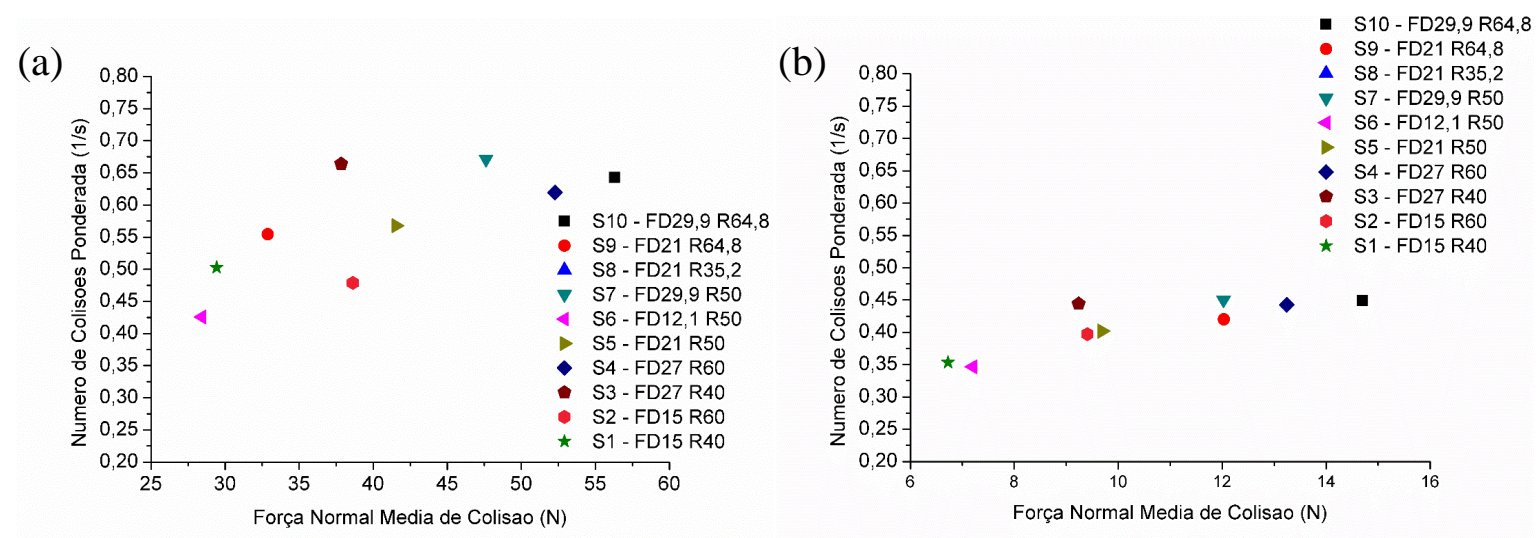

Conforme a Figura 3, observa-se que a força normal média de colisão das esferas de aço é maior quando comparada as esferas de porcelana. Em conformidade com a segunda Lei de Newton, quanto maior a massa de tais partículas no momento da colisão, maior será a força envolvida no impacto. Como as esferas de aço têm maior densidade do que as de porcelana, e por conta disso maior massa, o filme de material seco na superfície das partículas tende a apresentar um desprendimento facilitado devido às condições de impacto mais intenso verificados para as esferas de aço.

Figura 4 - Correlação obtida para Energia de Colisão: (a) Aço e (b) Porcelana.

(a)

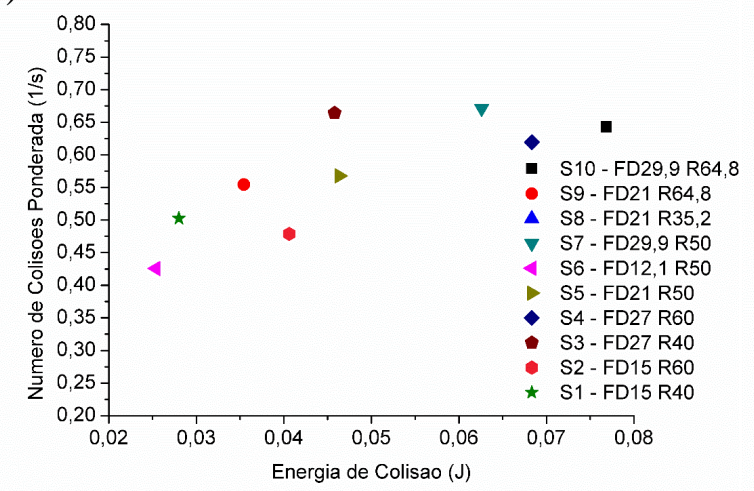

(b)

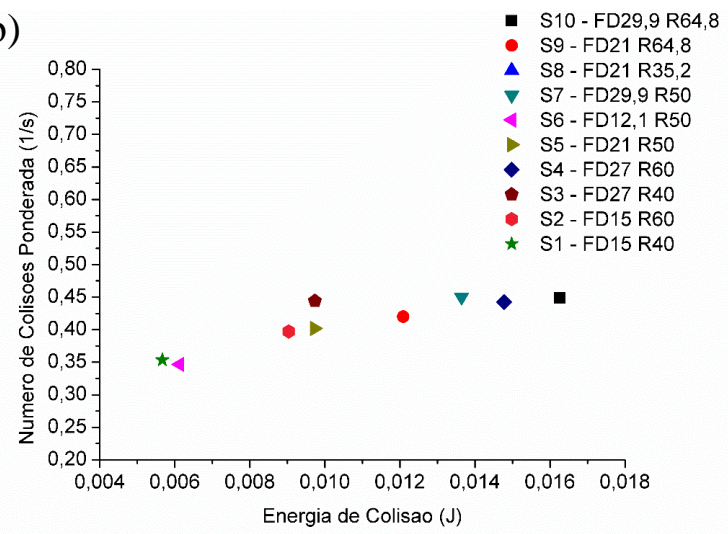

Como apresentado na Figura 4, observa-se que os valores de energia de colisão são expressivamente maiores nas esferas de aço. Isto se confirma por conta da relação da energia com a força de colisão citada anteriormente. A energia dissipada por ambas as partículas está ainda relacionada ao desprendimento do material da superfície após a colisão. Como a energia dissipada neste caso é maior quando comparado às esferas de porcelana, o desprendimento ocorre com maior intensidade com as esferas de aço. 


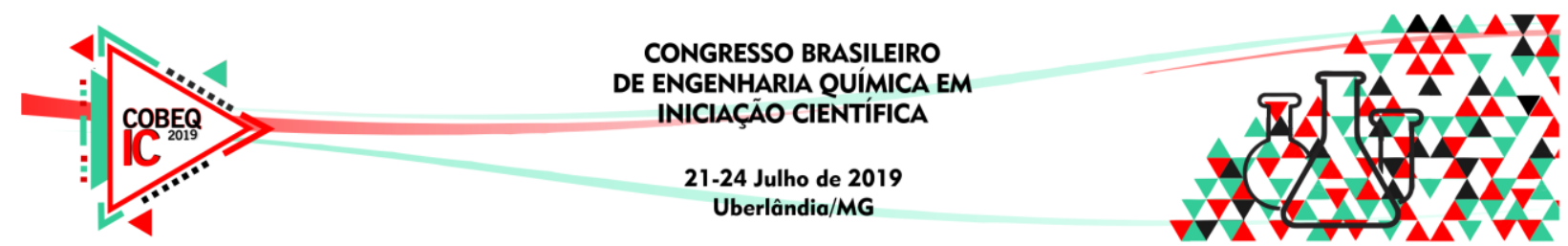

\section{CONCLUSÃO}

De acordo com os resultados obtidos, verificou-se que o uso de esferas de aço como inerte na secagem em tambor rotatório pode estar atrelado à um maior rendimento desse tipo de secador. As respostas verificadas pela aplicação da modelagem DEM levaram a melhor compreensão das interações entre as partículas e partículas-tambor no que diz respeito ao número de colisões, força e energia de colisão. Os resultados mostraram que, dentro das analises realizadas, o aumento do fator de enchimento resultou em um maior número de colisões entre os inertes. Além disso, verificou-se que as esferas de aço apresentaram colisão mais intensa e com maior desprendimento de energia, o que pode estar atrelado a um maior desprendimento superficial do material seco e, por consequência, maior rendimento do processo.

\section{REFERÊNCIAS}

BRACEY, R. J.; WEERASEKARA, N. S.; POWELL, M. S. Performance evaluation of the novel multi-shaft mill using DEM modelling. Minerals Engineering, v. 98, p. 251-260, 2016.

BRANDÃO, R. J. Estudo Da Segregação De Material Granular Abordagem Numérica Lagrangeana Abordagem Numérica Lagrangeana. [s.l.] Universidade Federal de Uberlândia, 2017.

BURJAILI, M. M. Desenvolvimento de um secador rotatório com recheio de inertes. [s.1.] Universidade Estadual de Campinas, 1996.

FREIRE, J. T. et al. A Review on Paste Drying with Inert Particles as Support Medium. Dry. Technol., v. 30, n. 4, p. 330-341, 2012.

HERTZ, H. R. Uber die Beruhrung fester elastischer Korper und Uber die Harte. Verhandlung des Vereins zur Beforderung des GewerbefleiBes, Berlin, p. 449, 1882.

MINDLIN, R. D.; DERESIEWICZ, H. Elastic Spheres in Contact under Varying Oblique Force. J. Appl. Mech., v. 20, p. 327-344, 1953.

MOURA, B. D. DE. Secador Rotatório com Alimentação Intermitente. [s.1.] Universidade Federal do Rio Grande do Norte, 2016.

MUJUMDAR, A. S. Handbook of industrial drying. [s.1.] CRC press, 2006.

PENG, B. Discrete Element Method (DEM) Contact Models Applied to Pavement Simulation. [s.l: s.n.]. 